\title{
Untersuchungen zur Verteilung des Klebstoffes im Bereich der Leimfuge mittels Neutronenradiographie und Mikroskopie
}

Online veröffentlicht: 3 November 2004

(C) Springer-Verlag 2004

Zusammenfassung Mittels mikroskopischer Betrachtung von angefärbten Dünnschnitten, Rasterelektronenmikroskopie sowie Neutronenradiographie wurde vergleichend das Eindringverhalten von Harnstoff-Formaldehydharz, Dispersionsklebstoff (PVA) und Polyurethan untersucht. Es konnten deutliche Unterschiede im Eindringverhalten der Klebstoffe nachgewiesen werden. Bei Harnstoffharz trat unter den gewählten Verklebungsbedingungen ein stärkeres Eindringen des Klebstoffes in das Holz auf. Mittels Neutronenradiographie konnte eine Quantifizierung der Leimverteilung erzielt werden. Mit dieser Methode gelang es, das Eindringen des Leimes sichtbar zu machen, und Leimfuge und Holz zu trennen. Es sind deutliche Unterschiede im Bereich FrühholzSpätholz erkennbar.

\section{Experiments on the distribution of adhesives close to the glue joint by neutron radiography and microscopy}

Abstract The permeation behaviour of different types of adhesives (urea-formaldehyde resin, dispersion glue (PVA) and Polyurethane) was investigated by light microscopy, scanning electron microscopy and neutron radiography. Clear differences in the permeation behaviour of the adhesives could be determined. Urea resin showed under the chosen conditions the strongest permeation of wood. The glue allocation was quantified and the permeation visualised by means of neutron radiography. By this a differentiation between wood and the glue joint was

P. Niemz $(\bowtie) \cdot$ D. Mannes

Institut für Baustoffe,

ETH Hönggerberg, HIF E 25.2,

8093 Zürich, Schweiz

E-Mail: niemz@ibwk.baug.ethz.ch

E. Lehmann · P. Vontobel

Paul Scherrer Institut,

Villigen, Schweiz

S. Haase

TU Dresden,

Dresden, Deutschland possible. The penetration depth of the glue was conspicuously different between spring wood and late wood areas.

\section{Einleitung}

In zunehmendem Maße wird die Klebverbindung in der Holzindustrie eingesetzt. Für die Festigkeitsausbildung und die Wirtschaftlichkeit der Verklebung sind auch das Eindringverhalten des Klebstoffes und die Fugendicke von Bedeutung. Für deren Untersuchung stehen verschiedene mikroskopische Methoden zur Verfügung (Zeppenfeld 1991, Wagenführ 1966). Umfangreiche Arbeiten zum Eindringverhalten von PUR Klebstoffen in das Holz wurden von Schirle und Mitarbeitern (2002) durchgeführt. Dabei erfolgte die Zugabe eines Fluoreszenzmittels. Die Betrachtung der Leimfuge erfolgte im Auflicht mittels Auflicht-Fluoreszenz. Dabei wurde festgestellt, dass der Klebstoff stärker in das Frühholz eindringt. Die Eindringtiefe lag bei Fichtenholz und einem aufgebrachten Leimtropfen aus PUR im Frühholz bei $56 \mu \mathrm{m}$, im Spätholz bei $22 \mu \mathrm{m}$. Bei unter Praxisbedingungen verklebten Leimfugen betrug bei PUR Klebstoff mit Primer die Eindringtiefe im Frühholz $150 \mu \mathrm{m}$, im Spätholz $70 \mu \mathrm{m}$. Gut erkennbar waren auch Luftblasen in der Leimfuge des PUR- Klebstoffes. Weiterführende Literatur zum Eindringverhalten des Klebstoffes in das Holz ist auch in Dunky und Niemz (2002) vorhanden. Gindel, Dessipri und Wimmer (2002) untersuchten das Eindringen von MUF Harzen in die Zellwand von Fichtenholz mittels UV-Spektroskopie. Sie konnten Melamin in der Zellwand nachweisen. Der Melaminanteil in der Zellwand betrug $6,2 \%$.

Im Rahmen der vorliegenden Arbeit wird die Methode der Neutronenradiographie und -tomographie zur Ermittlung des Eindringverhaltens von verschiedenen Klebstoffen erstmals getestet. Vergleichend werden die Rasterelektronenmikroskopie und die klassische Durchlichtmikroskopie angewandt. Die Grundlagen der Anwendung der Neutronenradiographie für holztechnische und holz- 
Tabelle 1 Gewählte Methodik zum Anfärben

Table 1 Staining methods employed

\begin{tabular}{llll}
\hline Klebstoffe & Basisprodukt & Nachweismittel & Reaktion \\
\hline Miracol & $\begin{array}{l}\text { Vinylester-Homopolymer } \\
\text { (Dispersionsklebstoff) }\end{array}$ & Jodjodkalium + Natriumthiosulfat-lösung & Leim: hellrot \\
Placol & $\begin{array}{l}\text { Harnstoff-Formaldehyd-Harz } \\
\text { (Polykondensationsklebstoff) }\end{array}$ & Iodjodkalium + Natriumthiosulfat-lösung & Leim: gelb \\
Mirapur & $\begin{array}{l}\text { Polyurethan-Praepolymer } \\
\text { (Polyurethanklebstoff) }\end{array}$ & Iodjodkalium + Natriumthiosulfat-lösung & Holz: unverfärbt \\
& & & Leim: verschiedene Rotfärbungen \\
& & & Holz : unverfärbt \\
\hline
\end{tabular}

physikalische Fragestellungen wurden von Lehmann et al. (2001), Lehmann et al. (2002a, b), Niemz et al. (2002); sowie Vontobel et al. (2002) ausführlich beschrieben, so dass diese hier nicht nochmals behandelt werden.

In den bisherigen Arbeiten wurden im wesentlichen die Abbildung von Werkstoffstrukturen und die Aufnahme bzw. Abgabe von Wasser durch Sorption und kapillare Transportprozesse geprüft. Aufbauend auf den bisherigen Erfahrungen wird nachfolgend getestet, inwieweit Klebstoff bei der Verleimung in das Holz eindringt. Dabei wurden zunächst lediglich unterschiedliche Klebstoffe bei konstanten Verleimungsbedingungen geprüft, um methodisch die Möglichkeiten und Grenzen abzuschätzen. Vergleichend wurde konventionelle Methoden (Anfärben, Rasterelektronenmikroskopische Aufnahmen) verwendet.

\section{Versuchsmaterial und Methodik}

\subsection{Versuchsmaterial}

Es wurden unter Normbedingungen aus Buche verklebte Prüfkörper der Abmessungen $50 \mathrm{~mm} * 20 \mathrm{~mm} * 5 \mathrm{~mm}$ verwendet. Als Klebstoff dienten:

- Miracol (Dispersionsklebstoff)

- Placol (Harnstoffharz)

- Mirapur (Polyurethan)

der Fa. Geistlich, Zürich.

2.2 Untersuchungen an angefärbten Dünnschnitten mittels Auflichtmikroskop

Für die Aufnahmen wurde eine Binokularlupe Olympus SZX9 mit Digitalkamera Olympus CAMEDIA C-Z verwendet.

Aus dem verleimten Holz wurden mittels Schlittenmikrotom zunächst Dünnschnitte von $50 \mu \mathrm{m}$ Dicke angefertigt. Um die für den Schnitt erforderliche Weichheit des Materials zu erhalten, erfolgte zunächst eine Lagerung der Proben im Wasserbad unter Vakuum. Durch die dabei im Holz auftretenden Quellvorgänge kann es zu gewissen Beeinträchtigungen der Leimfuge gekommen sein.
Die fertigen Dünnschnitte wurden anschliessend mit den in Tabelle 1 aufgeführten Indikatorlösungen (Zeppenfeld (1991)) angefärbt.

\subsection{Rasterlektronenmikroskopie}

Die zu untersuchenden Proben wurden auf Würfelformat mit $5 \mathrm{~mm}$ Kantenlänge zugeschnitten und die Oberfläche anschliessend mit einem Schlittenmikrotom geglättet. Um eine Veränderung der Klebstoffstruktur bei Wassereinfluss zu verhindern, wurden die Proben trocken geschnitten. Dadurch konnte die Schnittfläche nicht an allen Stellen absolut sauber geschnitten werden, so dass teilweise Haarrisse und kleine Ausbrüche auftraten. Anschließend wurden die Proben schonend im Trockenschrank darrgetrocknet. Die darrtrockenen Proben wurden danach auf vorbereitete Probenhalter geklebt und mit Gold besputtert. Die elektronenmikroskopischen Untersuchungen erfolgten mit einem Rasterelektronenmikroskop LEO 435 Vpi (Zeiss/Leitz/Cambridge).

\subsection{Neutronenradiographie und Tomographie}

Die Neutronenradiographie beruht auf dem Prinzip der Transmissionsmessung. Ausgehend von einer Quelle werden Neutronen durch einen Kollimator auf die Proben geleitet. Die Probe schwächt den Neutronenstrahl je nach Beschaffenheit und atomarer Zusammensetzung gemäß dem exponentiellen Abschwächungsgesetz und ein 2-dimensionaler Neutronendetektor registriert die transmittierten Neutronen. Die Messdaten z.B. in Form von 16bit CCD Bildern werden über Grauwertstufen analysiert. Der entscheidende Faktor ist der Neutronenschwächungskoeffizient, der zu untersuchenden Stoffe. Insbesondere Wasserstoff kann mit Neutronen gut detektiert werden. Einerseits lässt sich aus Abschwächungsprofilen die Klebstoffverteilung gemittelt über die Probe in Neutronenstrahlrichtung ableiten, andererseits kann mit einer Tomographiemessung die räumliche Verteilung des Klebstoffes in der Probe bestimmt werden (Abb. 1).

Obwohl Holz nur zu ca. 6\% aus Wasserstoff besteht, beruhen etwa $80 \%$ der Strahlabschwächung auf der Wechselwirkung der Neutronen mit den Wasserstoffkernen. Dadurch ist das Verfahren auf Probendicken bis zu $5 \mathrm{~cm}$ in Strahlrichtung beschränkt. Zusätzlich in die Holzstruktur eingebrachtes Wasser bzw. durch Trocknung 


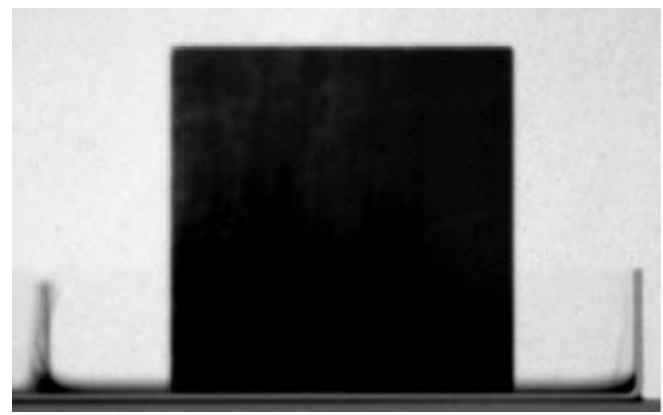

Abb. 1 Transmissionsradiographieaufnahme einer Holzprobe (Fichte) während eines Aufsaugversuches; links: originale, unreferenzierte Aufnahme, rechts: Referenzierung zum trockenen Ausgangszustand-die Netto-Verteilung der Flüssigkeit wird sichtbar

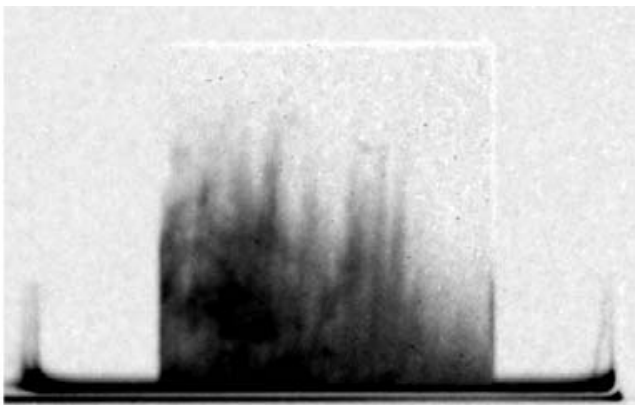

Fig. 1 Transmission radiograph of a wood sample (spruce) during an absorption test; left side: original radiograph, not referenced; right side: referencing to the dry initial state-the net-allocation of the fluid can be seen
Abb. 2 Analyse der Homogenität der Klebeverbindung bzw. der Leimverteilung entlang der Klebfuge, ermittelt aus den Radiographieaufnahmen senkrecht zur Leimfuge (Beispiel Placol, 8 Proben)

Fig. 2 Homogeneity analysis of the adhesive joint resp. the glue. Distribution along the adhesive joint; data ascertained by radiography perpendicular to the adhesive joint (example: Placol, 8 samples)
Dichteverlauf entlang der Klebstofffuge

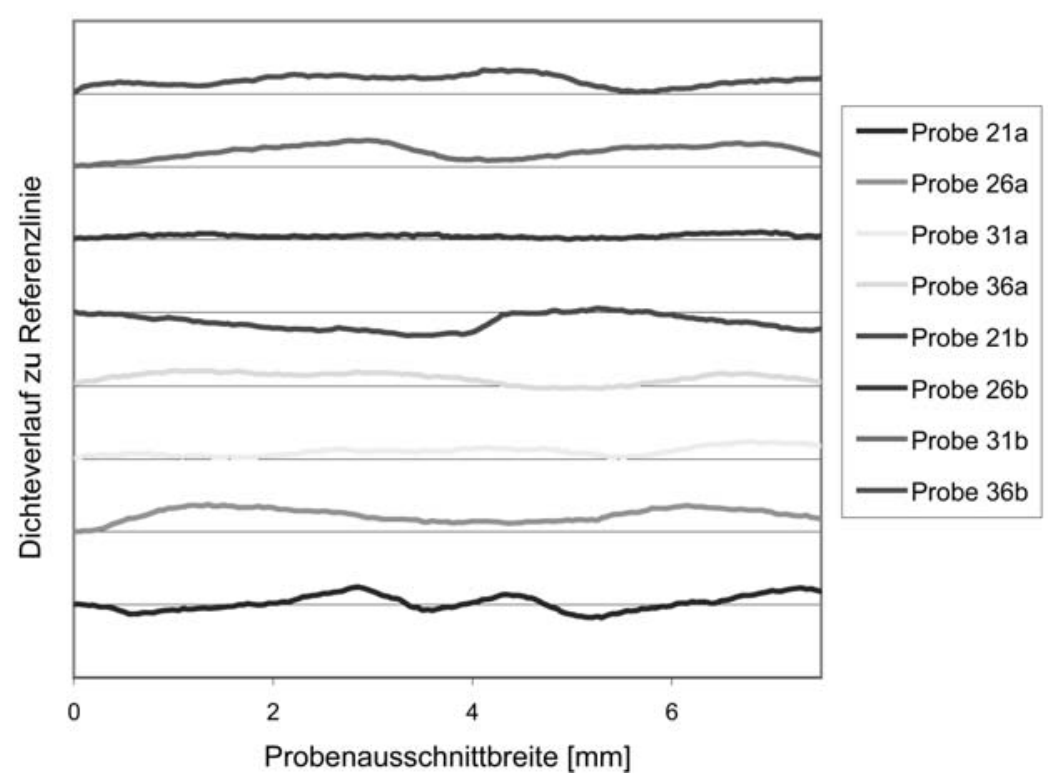

entferntes Wasser kann dennoch sehr empfindlich nachgewiesen werden, insbesondere, wenn Referenzverfahren zum Ausgangszustand der Proben angewendet werden. Ein Beispiel dafür ist in Abb. 2 gegeben.

Im Falle der Leimfugen konnte vorteilhaft die Tatsache genutzt werden, dass alle Leime einen hohen Wasserstoffgehalt aufweisen und sich deutlich von der Holzstruktur in den Radiographieaufnahmen unterscheiden.

Die Versuche wurden an der Neutronen-TransmissionRadiographiestation NEUTRA am Paul Scherrer Institut (PSI) in Villigen/Schweiz durchgeführt (http://neutra. web.psi.ch/). Zum Einsatz kamen digitale Neutronendetektionssysteme (Szintillator mit CCD-Kamera, Imaging Plates), die eine direkte quantitative Auswertung ermöglichten.

Es wurden 3 verschiedene Testserien durchgeführt:

\subsubsection{Transmissionsversuch 1}

Die Proben wurden zunächst auf $25 \mathrm{~mm}$ Dicke reduziert, d.h. entlang der Längsachse halbiert, die Ränder wurden geschliffen. Dann wurden die Proben in Gruppen zu je 12 Stück mittels Neutronenradiographie vermessen. Die Proben wurden dabei so ausgerichtet, dass sich die Klebefuge exakt parallel zum Neutronenstrahl befand. Als Detektor wurden zum einen Neutronen empfindliche Bildplatten der Firma Fuji mit $50 \mu \mathrm{m}$ Ortsauflösung und zum anderen ein Neutronen sensitiver Szintillator $(6 \mathrm{LiF} /$ $\mathrm{ZnS}: \mathrm{Ag}$ ) zusammen mit einer peltiergekühlten CCD Digitalkamera verwendet. Die Ortsauflösung liegt bei beiden Methoden bei ca. $100 \mu \mathrm{m}$. Für die weitere Analyse der Daten der Digitalkamera wurden noch Referenzaufnahmen ohne Proben bzw. ohne Neutronenstrahl angefertigt. Nachfolgend wurden mittels digitaler Bildbearbeitung die Untersuchungsergebnisse in folgenden Schritten bearbeitet. 
1. Normierung der Daten auf den Hintergrund (Hellfeld und Dunkelfeld) und Umrechnung der Messwerte in Absorptionswerte

2. Verschiebung der Kurven so, dass die Klebefugen aller Proben an der gleichen Position liegen

3. Ausschneiden der Bereiche der Klebefugen um die Datenmenge zu reduzieren

4. Teilung der Kurven in der Klebefuge, um den unterschiedlichen Holzdichten und den damit verbundenen verschiedenen Klebstoffverteilungen gerecht zu werden. Spiegelung der Kurvenstücke um eine bessere Darstellung zu erhalten.

5. Normierung der Kurven auf den Absorptionswert des Holzes

6. Mittelwertbildung der Probenwerte der jeweiligen Klebstoffsorten

7. Integralbildung der Klebestoffverteilungskurven um die, im Endzustand, vorhandene Klebstoffmenge zu errechnen

8. Bestimmung der prozentualen Klebstoffverteilungskurven
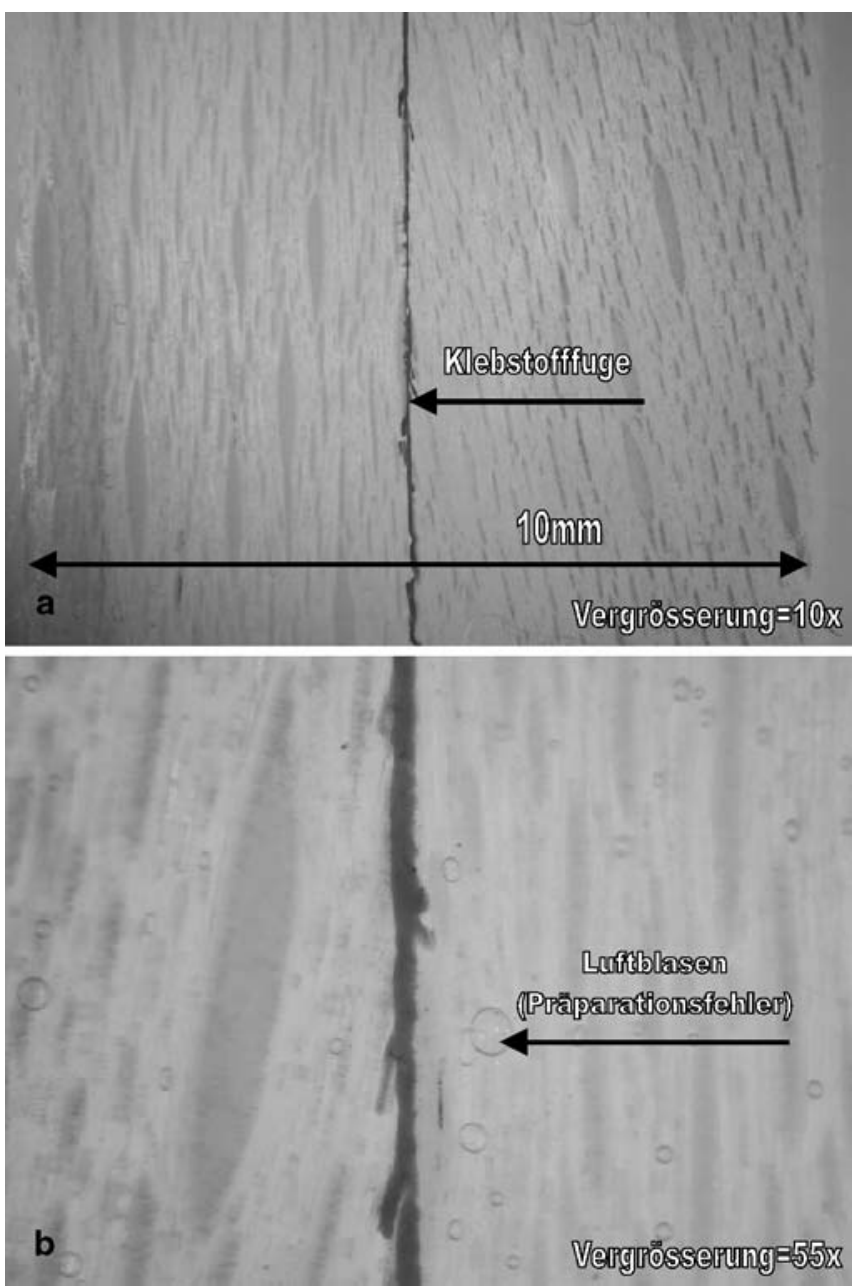

Abb. 3a,b Dünnschnittaufnahmen des mit Dispersionsklebstoff verleimten Holzes. a 10x; b $55 \times$

Fig. 3a,b Micrographs of a wood sample glued with dispersion adhesive. a $10 \times$; b $55 x$

\subsubsection{Transmissionsversuch 2}

Die Proben von Versuchsaufbau 1 wurden um $90^{\circ}$ gedreht, so dass sich der Neutronenstrahl senkrecht zu der Klebstofffuge befand. Die Analyse erfolgte in dieser Versuchsreihe nur mit Image-Platten. Die nachfolgende Auswertung wurde durchgeführt:

1. Normierung der Daten auf den Hintergrund und Umrechnung der Messwerte in Schwächungskoeffizienten

2. Anpassen der normierten Profile

3. Darstellung der Kurven übereinander mit je einer Referenzgeraden
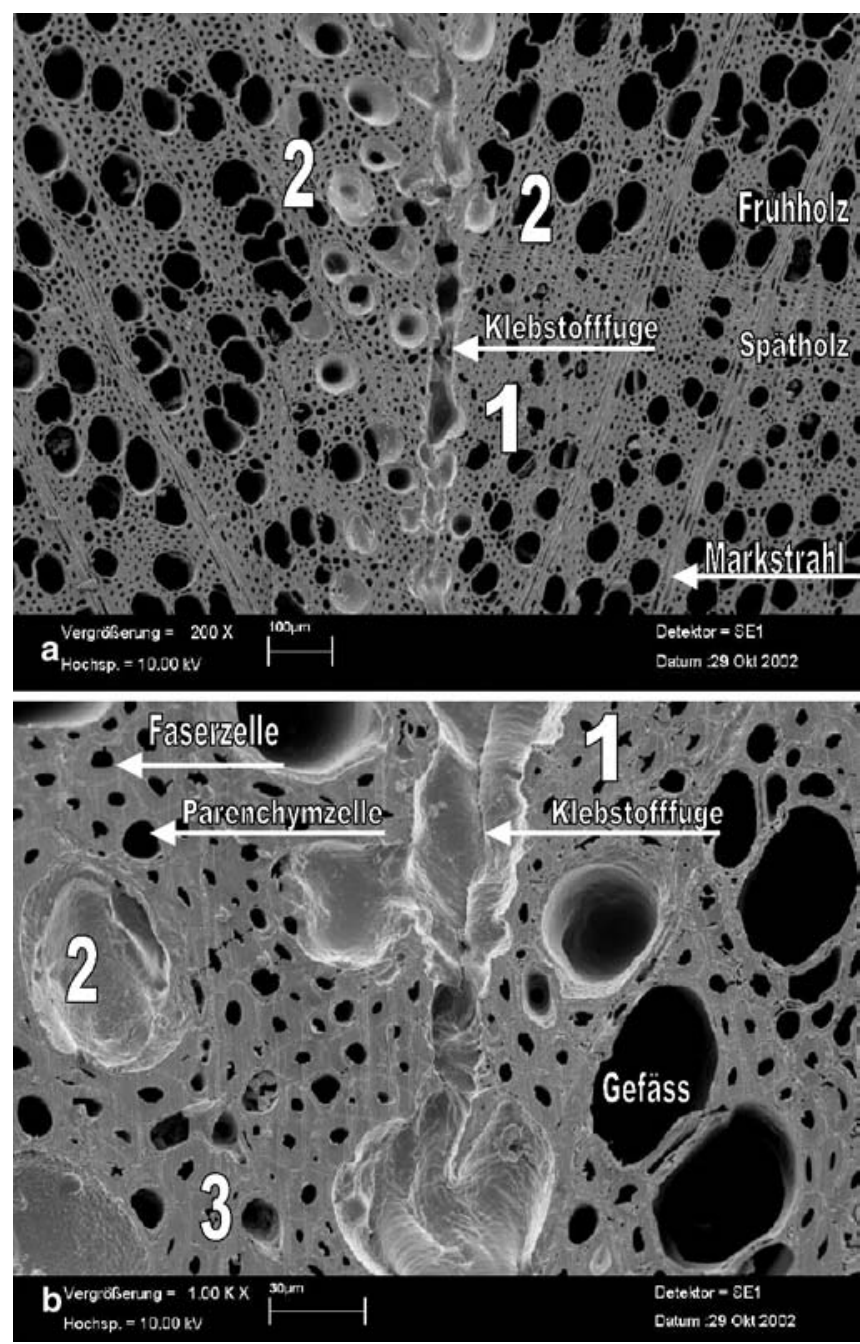

Abb. 4a,b Rasterelektronenmikroskopische Aufnahmen des mit Dispersionsklebstoff verleimten Holzes. a 200x; b 1000x. 1 Klebstofffuge, Ausbrüche durch Präparation bedingt; 2 Klebstoff in angrenzenden Gefäßen; 3 Faserzellen und Parenchymzellen ohne eingedrungenen Klebstoff

Fig. 4a,b Scanning electron micrographs of a wood sample glued with dispersion adhesive. a 200×, b 1000×. 1 Adhesive joint; chunking due to preparation, 2 adjacent vessels filled with adhesive, 3 fibres and parenchyma cells without permeating adhesive 


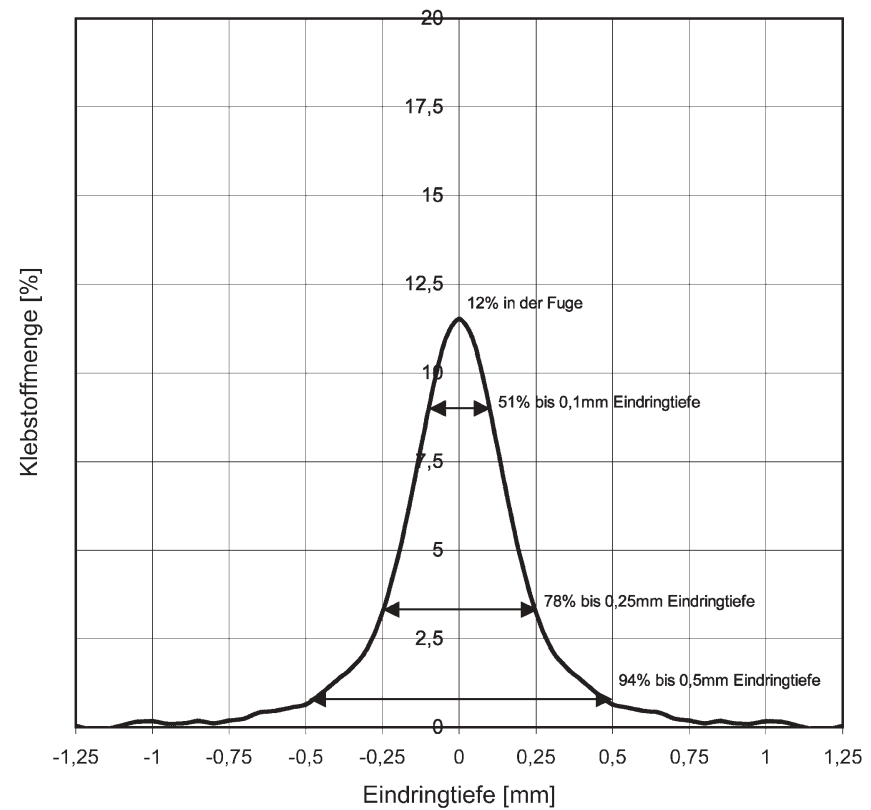

Abb. 5 Relative Verteilung der Klebstoffmenge senkrecht zur Leimfuge, bestimmt mittels Neutronenradiographie bei Verklebung mit Dispersionsklebstoff

Fig. 5 Relative distribution of the adhesive (dispersion glue) vertical to the adhesive joint; determined by neutron radiography

Mit dieser Darstellung (Abb. 2) konnten die Inhomogenitäten der Leimfugen entlang der Proben analysiert werden.

\subsubsection{Tomographie}

Um die dreidimensionale Verteilung des Klebstoffes in der Klebefuge sichtbar zu machen wurde eine ausgewählte Probe mit Neutronentomographie untersucht. Dazu wurde eine nach Früh- und Spätholzanteil repräsentative mit Placol (Harnstoffharz) verklebte Probe der Abmessung $10 \mathrm{~mm} \times 10 \mathrm{~mm} \times 50 \mathrm{~mm}$ herangezogen. Diese Probe wurde auf einem Drehtisch im Neutronenstrahl fixiert und in kleinen Winkelschritten von je $0,75^{\circ}$ von $0^{\circ}$ auf $180^{\circ}$ gedreht. Dabei wurden an der sich drehenden Probe von allen Seiten Transmissionsaufnahmen angefertigt. Diese Daten wurden anschliessend mittels Tomographie-Rekonstruktion (d. h. ,filtered back projecti-

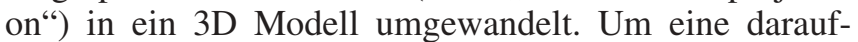
folgende räumliche Analyse der Struktur bzw. der Materialenverteilung des Probekörpers zu ermöglichen, wurden die Daten zu Videosequenzen weiter verarbeitet oder mittels einem 3D Visualisierungsprogramm in Holz- und Klebstoffanteil segmentiert.
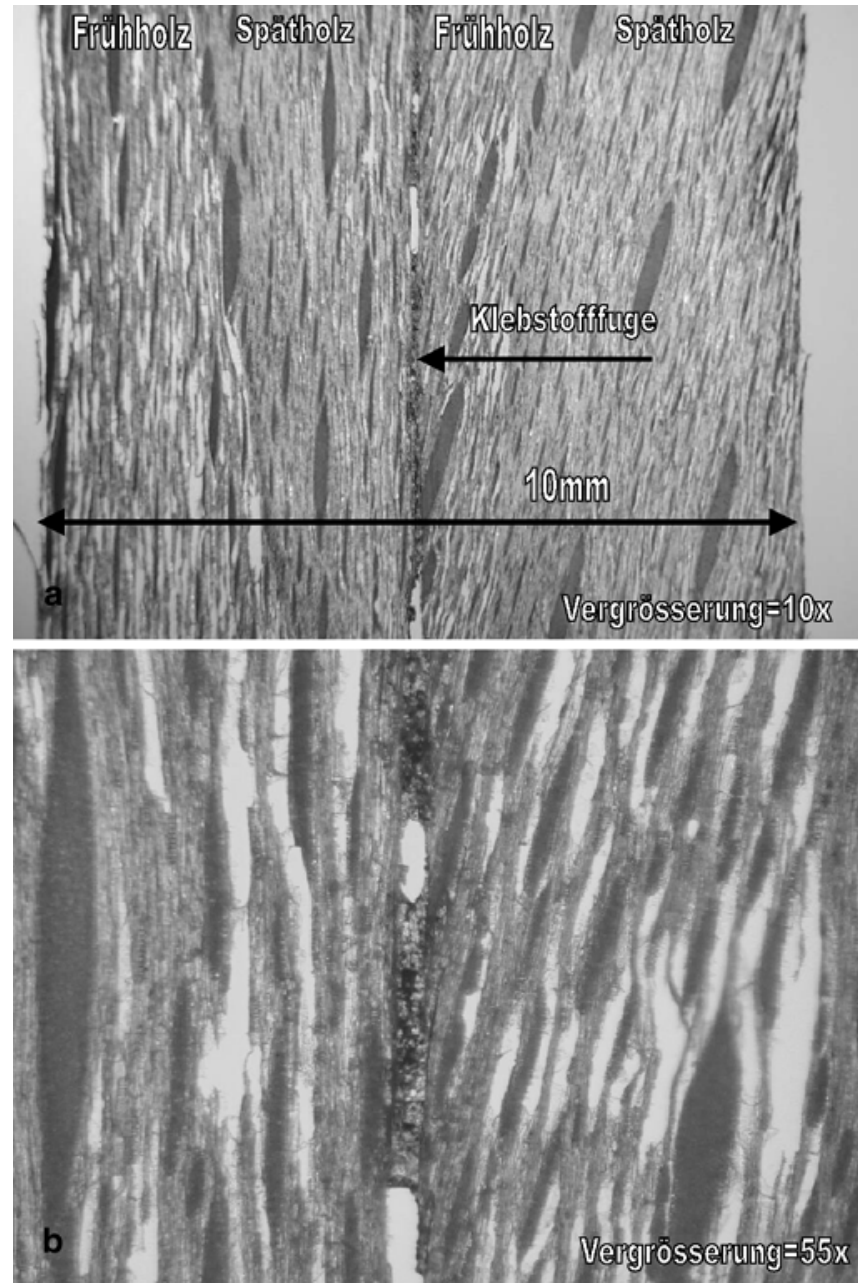

Abb. 6a,b Dünnschnittaufnahmen des mit Polyurethanklebstoff (PUR) verleimten Holzes. a 10x; b 55x

Fig. 6a,b Micrographs of a wood sample glued with polyurethane adhesive (PUR). a 10x; b 55x

\section{Versuchsergebnisse}

\subsection{Dispersionsklebstoff}

Abbildung 3 zeigt in unterschiedlicher Vergrößerung eine Transmissionsaufnahme der Leimfuge. Der Dispersionsklebstoff ist gut an der roten Färbung erkennbar (Abb. 3a). In Abb. 3b ist bei 55facher Vergrößerung recht gut das Eindringen des Klebstoffes in das Holz ersichtlich.

In den elektronenmikroskopischen Aufnahmen sind die Struktur des Klebstoffes sowie in besonderem Masse das Eindringen des Leimes in bestimmte Strukturelemente des Holzes deutlich zu erkennen. Einzelne Gefäße, die an die Leimfuge angrenzen sind vollständig mit Klebstoff gefüllt (Abb. 4a und b). Im Gegensatz zu den mikroskopischen Verfahren erlaubt die Neutronenradiographie eine Quantifizierung der Klebstoffverteilung im Holz (Abb. 5). Die Ergebnisse der Neutronenradiographie stimmen mit denen der Transmissions- und der Rasterelektronenmikroskopie überein. 

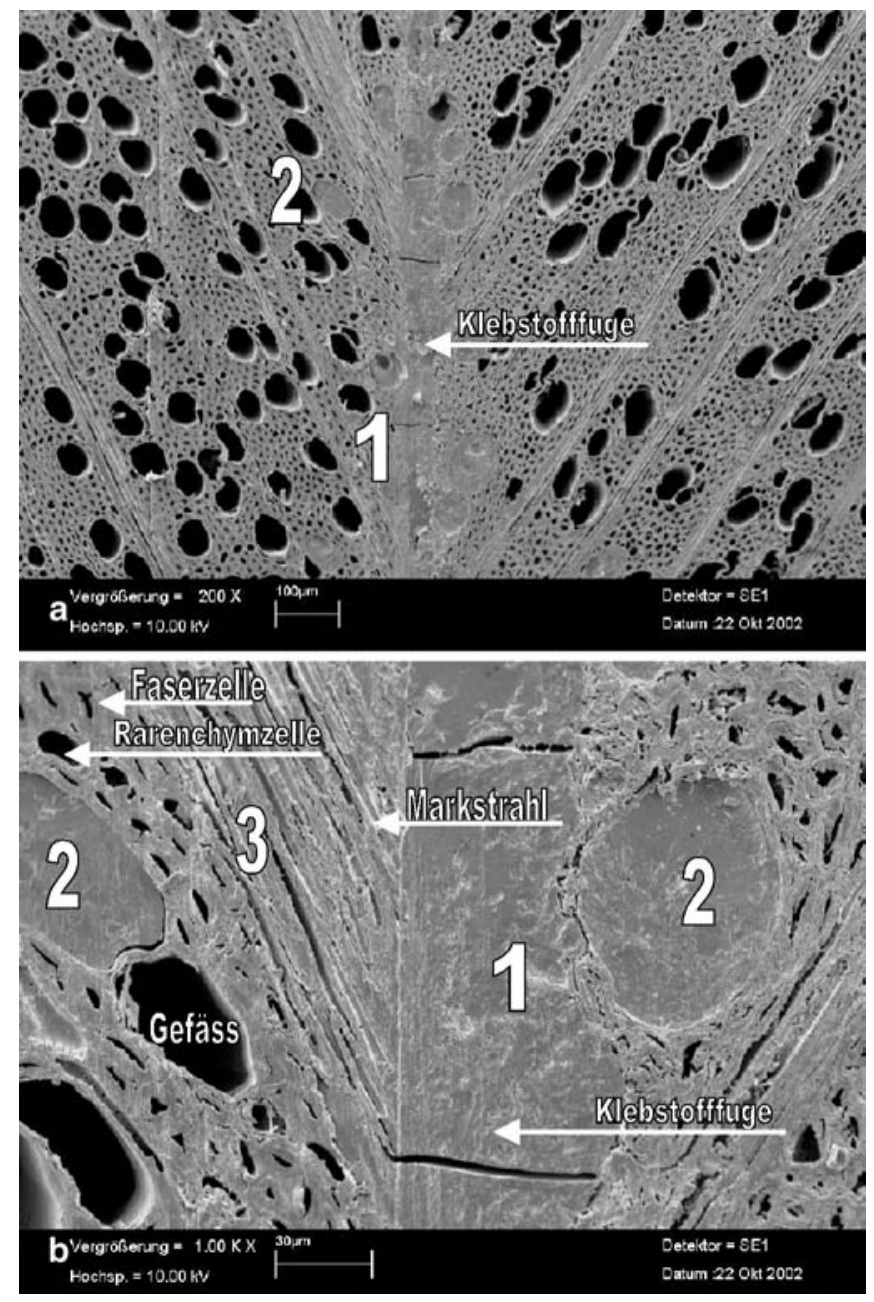

Abb. 7a,b Rasterelektronenmikroskopische Aufnahme des mit PUR verleimten Holzes. a 200x; b 1000×. 1 Klebstofffuge, Rissbildung vermutlich durch Präparation; 2 Klebstoff in angrenzenden Holzgefäßen sichtbar; 3 Faserzellen und Parenchymzellen und Markstrahlen ohne eingedrungenen Klebstoff

Fig. 7a,b Scanning electron micrograph of a wood sample glued with polyurethane adhesive (PUR). a 200x; b 1000×. 1 Adhesive joint; cracks due to preparation; 2 in adjacent vessels adhesive visible; 3 fibres, parenchyma cells and wood rays without permeating adhesive

\subsection{Polyurethan}

Die Klebfuge für die Transmissionsaufnahme ist gelblich braun gefärbt (Abb. 6). Der Klebstoff dringt nicht sehr stark in das Holz ein, Gefäße im Bereich der Leimfuge sind aber mit Leim gefüllt.

In den REM-Aufnahmen sind die Leimfuge sowie das Eindringen des Klebstoffes deutlich erkennbar (Abb. 7). Bei den in der Leimfuge auftretenden Rissen handelt es sich vermutlich um präparationsbedingte Artefakte. Abbildung 8 zeigt die mittels Neutronenradiographie ermittelte Eindringtiefe. Es ist eine deutliche Konzentration in der Leimfuge erkennbar.

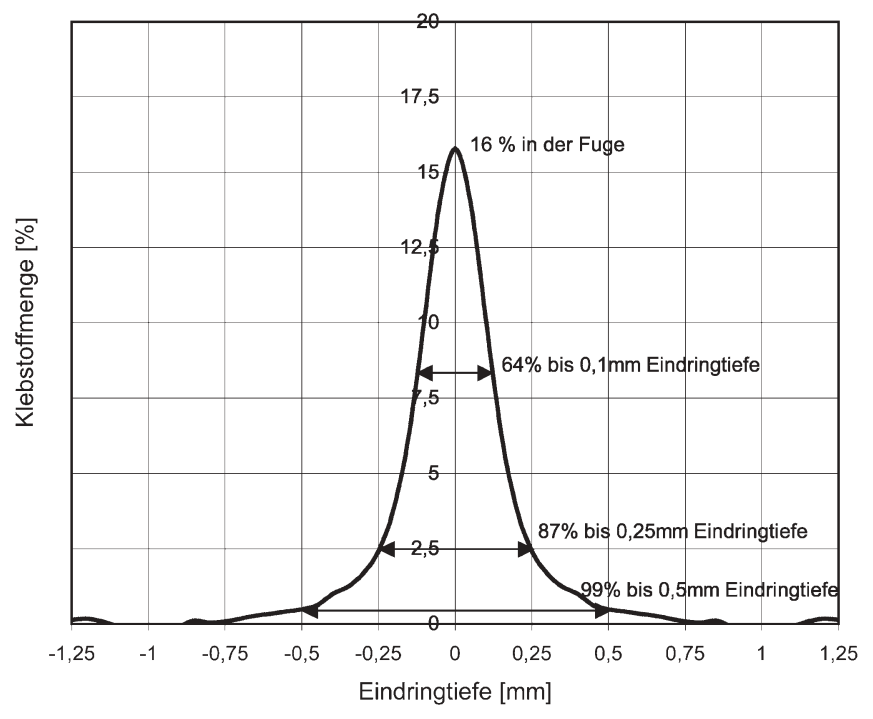

Abb. 8 Relative Verteilung der Klebstoffmenge senkrecht zur Leimfuge, bestimmt mittels Neutronenradiographie bei Verklebung mit PUR

Fig. 8 Relative allocation of the adhesive (PUR) vertical to the adhesive joint; determined by neutron radiography

\subsection{Harnstoffharz}

Abbildung 9 zeigt die Transmissionsaufnahmen der mit Harnstoffharz verklebten Proben. Deutlich ist das starke Eindringen des Klebstoffes in das Holz erkennbar. Der Klebstoff ist gelblich angefärbt. Die Gefäße und Parenchymzellen in der Umgebung der Klebfuge sind mit Leim gefüllt. Zusätzlich wurden Proben aufgenommen, bei denen nur ein einseitiger Klebstoffauftrag erfolgte (Abb. 9c), wobei deutliche Unterschiede im Eindringverhalten zu erkennen sind (vgl. Abb. 9b und c). Bei einseitigen Klebstoffauftrag zeigt die Seite, auf welcher der Klebstoff aufgetragen wurde, im Bereich der Grenzfläche eine deutlich höhere Leimkonzentration als die anderen Seite auf.

Dies spiegelt sich auch in den rasterelektronenmikroskopischen Aufnahmen sowie den mittels Neutronenradiographie bestimmten Eindringtiefen wieder (Abb. 10 und 11).

\subsection{Vergleich der Eindringtiefen} mittels Neutronenradiographie

Abbildung 12 sowie Tabelle 2 zeigen zum Vergleich das Eindringverhalten der 3 Klebstoffe mittels Neutronenradiographie. Es ist zu erkennen, dass Harnstoffharz am stärksten in das Holz $(0,75$ bis zu $1,25 \mathrm{~mm}$ in beiden Richtungen) eindringt. Dies deckt sich auch mit den mikroskopischen und elektronenmikroskopischen Aufnahmen. Das Eindringverhalten wird sehr stark durch die Feuchte, die Oberflächenrauhigkeit, die Viskosität des Klebstoffes und andere Parameter (z. B. auch Pressdruck) 

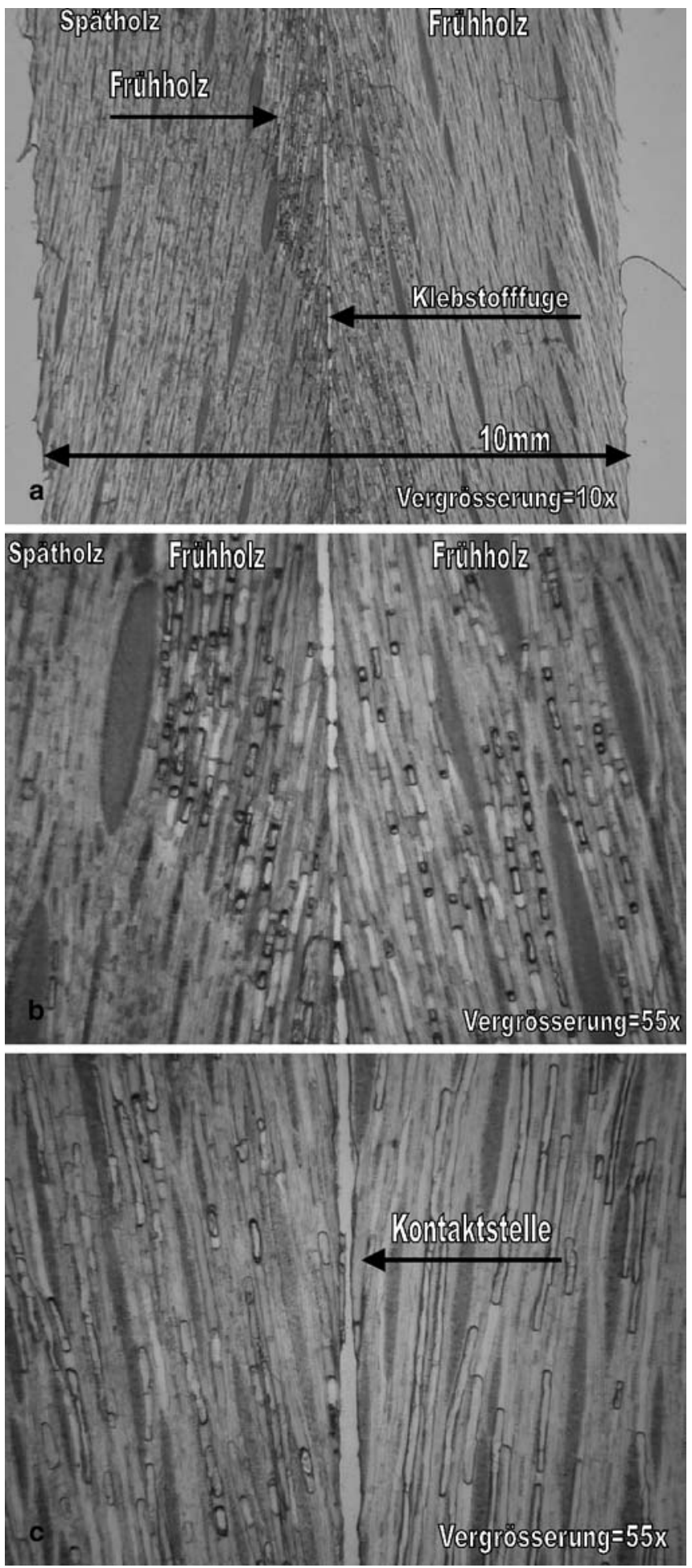

Abb. 9a-c Dünnschnittaufnahmen des mit Harnstoffharz verleimten Holzes. a 10x; b 55x, beidseitiger Klebstoffauftrag; c 55x; einseitiger Klebstoffauftrag

Fig. 9a-c Micrographs of a wood sample glued with urea resin. a 10x; b 55x; double-sided adhesive application; c 55x; one-sided adhesive application
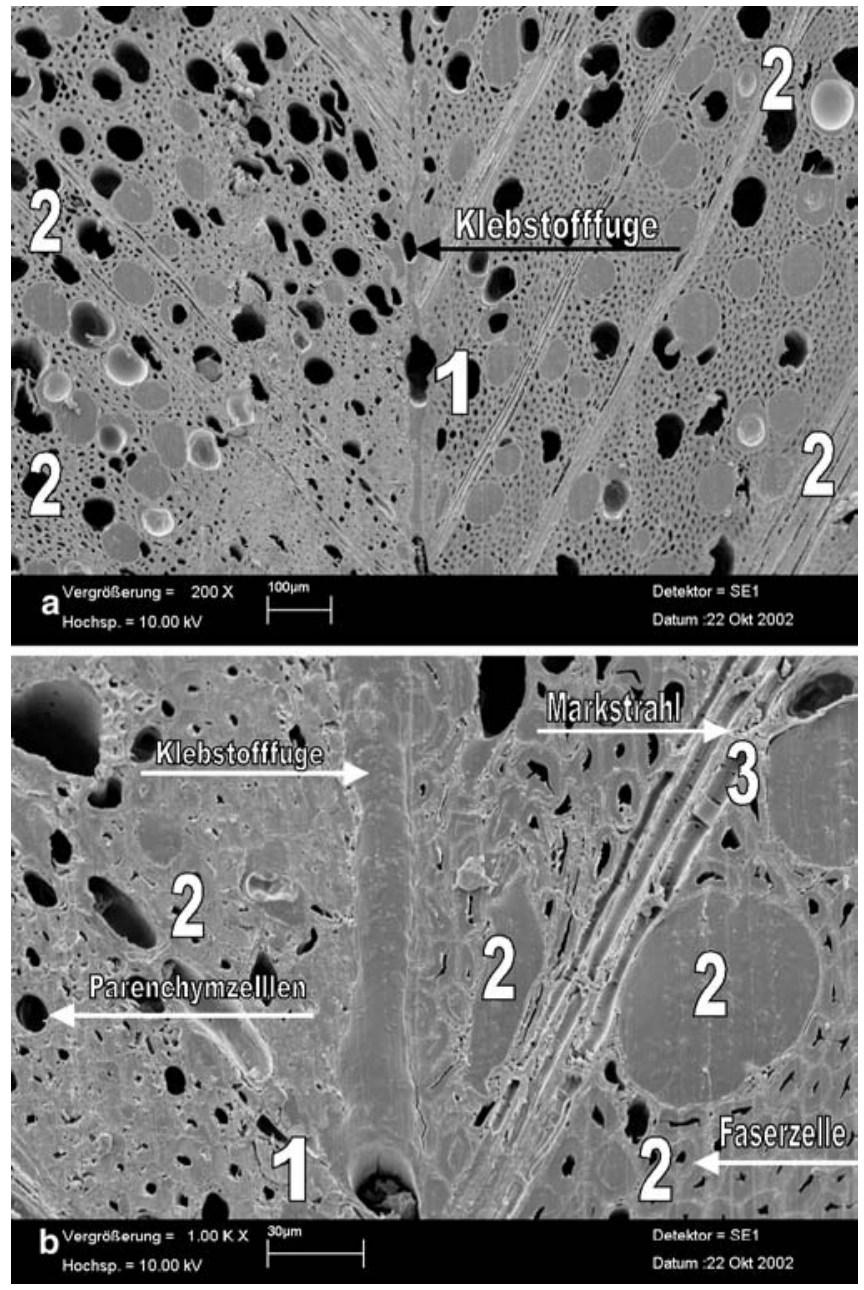

Abb. 10a,b Rasterelektronenmikroskopische Aufnahme des mit Harnstoffharz verleimten Holzes. a 200x; b 1000x. 1 Klebstofffuge; 2 Klebstoff in Gefäßen, Faserzellen und Parenchymzellen sichtbar; 3 Kein Klebstoff in Markstrahlen sichtbar

Fig. 10a,b Scanning electron micrograph of a wood sample glued with urea resin. a 200x; b $1000 \times$. 1 Adhesive joint; 2 visible adhesive in vessels, fibres and parenchyma cells; 3 no adhesive in wood rays

bestimmt. Hierzu sind weiterführende Arbeiten erforderlich.

\subsection{Neutronentomographie}

Dieser Datensatz wurde zu einer Videosequenz weiterverarbeitet. Abbildung 13 ist ein Einzelbild der Videosequenz. Der Klebstoff ist rot und das Holz ist gelblich dargestellt. Wie im Bild zu sehen ist, ist es möglich, Holz und Leim getrennt zu betrachten. Im Bild ist sehr deutlich die strangartige Ausbildung des Klebstoffes zu erkennen. Hier handelt es sich offensichtlich um Unterschiede im Eindringverhalten zwischen Früh- und Spätholz.

Die vorgestellten Methoden der Neutronenradiographie und -tomographie sind sehr geeignet, mit hoher Ortauflösung und gutem Kontrast die Verteilung von 
Abb. 11 Relative Verteilung der Klebstoffmenge senkrecht zur Leimfuge, bestimmt mittels Neutronenradiographie bei Verklebung mit Harnstoffharz Fig. 11 Relative distribution of the adhesive (urea resin) vertical to the adhesive joint; determined by neutron radiography

Abb. 12 Vergleich der Eindringtiefen der 3 Klebstoffe mittels Neutronenradiographie Fig. 12 Comparison of the permeation depth of the three adhesives determined by neutron radiography
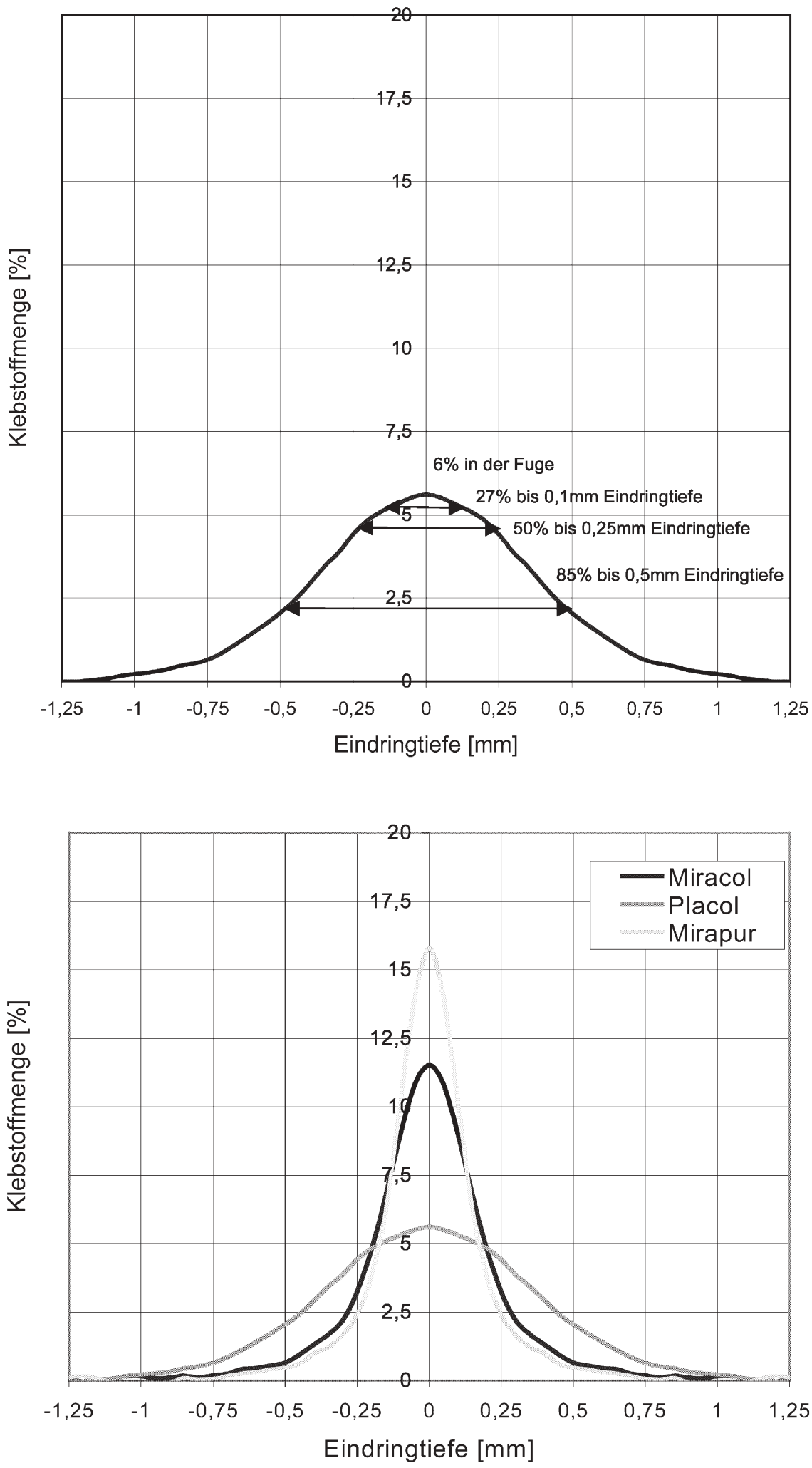

Klebstoffen in Holz zerstörungsfrei zu analysieren. Der Nachteil der Methode, bei den Untersuchungen in der Probendicke beschränkt zu sein, kann durch geeignete Wahl der Probengeometrien in vielen Anwendungsfällen umgangen werden.
Analoge Untersuchungen können für das Eindringverhalten von Imprägniermitteln, Harzen und Lacken durchgeführt werden. 
Tabelle 2 Mittels Neutronenradiographie bestimmte Verteilung des Klebstoffes in der Leimfuge Table 2 Glue distribution identified via neutron radiography

\begin{tabular}{|c|c|c|c|}
\hline & Miracol 6360 & Mirapur 9520 & Placol 4500 \\
\hline & [Dispersionsklebstoff] & [Polyurethanklebstoff] & [Polykondensationsklebstoff] \\
\hline \multicolumn{4}{|l|}{ Klebstoffverteilung [\%] } \\
\hline Von der Fuge bis $0,1 \mathrm{~mm}$ & 51 & 64 & 27 \\
\hline Von der Fuge bis $0,25 \mathrm{~mm}$ & 78 & 87 & 50 \\
\hline Von der Fuge bis $0,5 \mathrm{~mm}$ & 94 & 99 & 85 \\
\hline Klebfuge & - & Stellenweise luftblasenartige Löscher & - \\
\hline
\end{tabular}

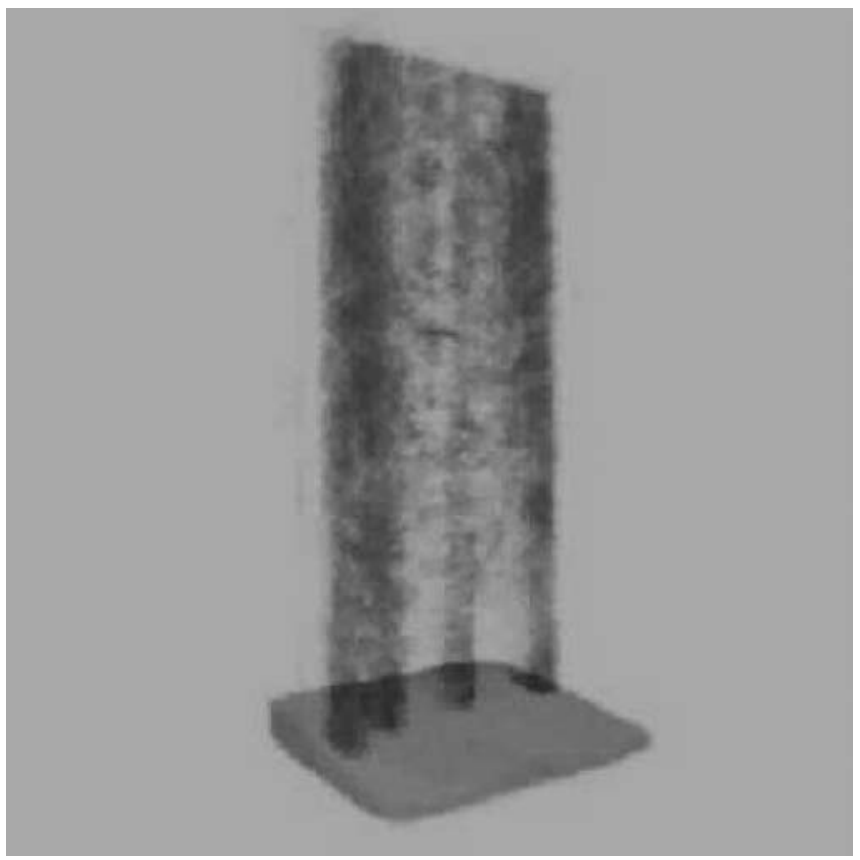

Abb. 13 Tomographie einer Verleimung mittels Harnstoffharz (Leimfuge rot, Holz gelblich)

Fig. 13 Tomography of an adhesion by urea resin (adhesive joint red, wood yellowish)

\section{Literatur}

Autorenkollektiv (1990) Holz-Lexikon. DRW Verlag, Stuttgart
Autorenkollektiv (1999) Holztechnik Fachkunde, 17. Aufl. Verlag Europa-Lehrmittel

Dunky M, Niemz P (2002) Holzwerkstoffe und Leime. Springer, Berlin Heidelberg New York

Gindel W, Dessipri E, Wimmer R (2002) Using UV-microscopy to study diffusion of melamine-urea-formaldehyd resin in cell walls of spruce wood. Holzforschung 56:103-107

Kucera LJ (1986) Einrichtungen und Methoden der biologischen Holzforschung. Institut für Wald- und Holzforschung ETHZ, Fachbereich Holzkunde und Holztechnologie, Zürich

Lehmann E, Vontobel P, Niemz P (2001) Anwendung der Methode der Neutronenradiographie zur Analyse von Holzeigenschaften. Holz Roh- Werkstoff 59:463-471

Lehmann E, Vontobel P, Usbeck T, Niemz P (2002) The potential of neutrons for the study of wood properties. 7. Weltkongress Neutroneradiographie, Rom

Lehmann E, Vontobel P, Niemz P, Usbeck T, Haase S (2002) New aspects for the wood analysis with neutron imaging methods. Jahresbericht, Paul Scherrer Institut, Villigen

Niemz P, Lehmann E, Vontobel P, Haller P, Hanschke S (2002) Untersuchungen zur Anwendung der Neutronenradiographie zur Beurteilung des Eindringverhaltens von Wasser in Eckverbindungen aus Holz. Holz Roh- Werkstoff 60:118-126

Schirle MA, Künniger T, Fischer A, Richter K (2002) Charakterisierung und Optimierung der Holzverklebung mit 1 Komponenten Polyurethan (1K-PUR) Klebstoffen. KTI Abschlussbericht 4126.1, EMPA, Abteilung Holz, Dübendorf

Vontobel P, Lehmann E, Niemz P, Usbeck T (2002) First neutron tomography investigations of wood-problems and their pragmatical solution. 13. Symposium für Zerstörungsfreie Prüfung, Berkeley, 19.-21.8.

Wagenführ R (1966) Anatomie des Holzes. Fachbuchverlag, Leipzig

Zeppenfeld G (1991) Klebstoffe in der Holz- und Möbelindustrie. Fachbuchverlag, Leipzig 